\title{
SPATIALLY INDUCED GROUPS OF AUTOMORPHISMS OF CERTAIN VON NEUMANN ALGEBRAS $\left({ }^{1}\right)$
}

\author{
BY
}

\author{
ROBERT R. KALLMAN
}

\begin{abstract}
This paper gives an affirmative solution, in a large number of cases, to the following problem. Let $\mathscr{R}$ be a von Neumann algebra on the Hilbert space $\mathscr{H}$, let $G$ be a topological group, and let $a \rightarrow \varphi(a)$ be a homomorphism of $G$ into the group of *-automorphisms of $\mathscr{R}$. Does there exist a strongly continuous unitary representation $a \rightarrow U(a)$ of $G$ on $\mathscr{H}$ such that each $U(a)$ induces $\varphi(a)$ ?
\end{abstract}

0. Introduction. The following problem arises in quantum mechanics. Let $\mathscr{R}$ be a von Neumann algebra on the Hilbert space $\mathscr{H}, \mathscr{G}$ a topological group, and $a \rightarrow \varphi(a)$ a representation of $G$ into the group of $*$-automorphisms of $\mathscr{R}$. When does there exist a strongly continuous unitary representation $a \rightarrow U(a)$ of $G$ on $\mathscr{H}$ such that each $U(a)$ induces $\varphi(a)$ ? Roughly speaking, this question asks: Does a Hamiltonian exist? The main result of this paper is to prove the following theorem, which gives an affirmative answer to the above question under fairly general hypotheses.

THEOREM 0.1. Let $\mathscr{R}$ be a semifinite von Neumann algebra on the separable Hilbert space $\mathscr{H}$. Suppose that the commutant of $\mathscr{R}$ has no finite portion. Let $G$ be a locally compact group whose topology is second countable, and let $a \rightarrow \varphi(a)$ be a representation of $G$ as a group of center-fixing *-automorphisms of $\mathscr{R}$. Suppose that $a \rightarrow\langle\varphi(a)(T) x, y\rangle$ is continuous for all $T$ in $\mathscr{R}$ and $x, y$ in $\mathscr{H}$. Then there exists $a$ strongly continuous unitary representation $a \rightarrow U(a)$ of $G$ on $\mathscr{H}$ such that $\varphi(a)(T)$ $=U(a) T U\left(a^{-1}\right)(a$ in $G, T$ in $\mathscr{R})$.

One cannot omit the hypothesis that the commutant of the properly infinite portion of $\mathscr{R}$ is also properly infinite, for there exists a $\mathrm{II}_{\infty}$ factor $\mathscr{S}$, with $\mathscr{S}^{\prime}$ finite, which possesses nonspatial *-automorphisms (see Kadison [6]). I do not know if an analogue of Theorem 0.1 is true in case $\mathscr{R}$ has a portion of type III $_{\infty}$.

$\S 1$ contains a proof of Theorem 0.1 in case $\mathscr{R}$ is a factor. $\S 2$ and $\S 3$ are devoted to preliminary topics in direct integral theory and Borel structure theory of various objects. The proof of Theorem 0.1 is given in $\$ 4$ by combining the results of the previous three sections.

Received by the editors April 6, 1970 and, in revised form, July 27, 1970.

AMS 1968 subject classifications. Primary 4600; Secondary 4665.

Key words and phrases. von Neumann algebras, operator algebras, groups of automorphisms, quantum mechanics.

(1) Research supported in part by SAR/F-44620-67-C-00090.

Copyright (C) 1971, American Mathematical Society 
See the two books by Dixmier ([2] and [4]) for the basic notation and facts about von Neumann algebras and $C^{*}$-algebras used in this paper. Use Mackey [7] and Parthasarathy [10] as references for the theory of standard Borel spaces.

1. Some preliminary cases. We recall a few elementary facts about Hilbert algebras for the convenience of the reader. Let $\mathscr{A}$ be an associative algebra over the complex numbers with an involutory anti-automorphism $T \rightarrow T^{*}$. Let $\langle\cdot, \cdot\rangle$ be a positive definite inner product on $\mathscr{A} \times \mathscr{A}$, and let $\mathscr{H}$ be the Hilbert space completion of $\mathscr{A}$. Suppose $\mathscr{A}$ also satisfies the following: (1) $\langle S, T\rangle=\left\langle T^{*}, S^{*}\right\rangle$; (2) $\langle R S, T\rangle=\left\langle S, R^{*} T\right\rangle$; (3) the mapping $T \rightarrow S T$ is continuous for each $S$ in $\mathscr{A}$; (4) $\mathscr{A}^{2}$ is dense in $\mathscr{A}$. An $\mathscr{A}$ with the above properties is called a Hilbert algebra.

One may easily check that $T \rightarrow T S$ is also continuous for each $S$ in $\mathscr{A}$. Let $J$ be the unique conjugate linear isometry of $\mathscr{H}$ which extends $T \rightarrow T^{*}$. Let $L(S)(R(S))$ be the unique bounded operator on $\mathscr{H}$ which extends the mapping $T \rightarrow S T$ $(T \rightarrow T S)$ of $\mathscr{A}$ into $\mathscr{A}$. The mappings $S \rightarrow L(S), S \rightarrow R(S)$ are *-isomorphisms of $\mathscr{A}$ onto their ranges. One also has that $L(S)=J R(S) J(S \in \mathscr{A})$.

Denote by $L(\mathscr{A})(R(\mathscr{A}))$ the strong closure of the algebras generated by $L(S)$, $S \in \mathscr{A}(R(S), S \in \mathscr{A}) . L(\mathscr{A})$ and $R(\mathscr{A})$ are von Neumann algebras, $L(\mathscr{A})^{\prime}=R(\mathscr{A})$, $R(\mathscr{A})^{\prime}=L(\mathscr{A})$, and $L(\mathscr{A})=J R(\mathscr{A}) J$.

LEMMA 1.1. Let $G$ be a topological group, and let $a \rightarrow \varphi(a)$ be a representation of $G$ into the group of ${ }^{*}$-automorphisms of $\mathscr{A}$. Suppose that $a \rightarrow\left\langle\varphi(a)\left(T_{1}\right), T_{2}\right\rangle$ is continuous on $G$ for all $T_{1}, T_{2}$ in $\mathscr{A}$, and that there exists a continuous homomorphism $a \rightarrow \mu(a)$ of $G$ into the positive reals such that each $\mu(a) \varphi(a)$ preserves inner products on $\mathscr{A} \times \mathscr{A}$. Then there exists a strongly continuous unitary representation $a \rightarrow W(a)$ of $G$ on $\mathscr{H}$ such that $W(a) L(T) W\left(a^{-1}\right)=L(\varphi(a)(T))(a \in G, T \in \mathscr{A})$.

Proof. Let $W(a)$ be the linear operator on $\mathscr{A}$ defined by $W(a)(T)=\mu(a) \varphi(a)(T)$. Since $\|W(a)(T)\|=\|T\|, W(a)$ extends to an isometry of $\mathscr{H}$, which we also denote by $W(a) . W(a)$ is actually unitary since $W(a) W\left(a^{-1}\right)=W\left(a^{-1}\right) W(a)=I$. It suffices to show that $a \rightarrow W(a)$ is continuous in the weak operator topology, for the weak operator topology and the strong operator topology coincide on the group of unitaries. Easy estimates show that it suffices to prove that $a \rightarrow\langle W(a) x, y\rangle$ is continuous for all $x, y$ in some dense linear submanifold of $\mathscr{H}$. But if $x=T_{1} \in \mathscr{A}$ and $y=T_{2} \in \mathscr{A},\langle W(a) x, y\rangle=\mu(a)\left\langle\varphi(a)\left(T_{1}\right), T_{2}\right\rangle$, which by assumption is a continuous function of $a$. Finally, if $S$ and $T$ are in $\mathscr{A}, W(a) L(S) W\left(a^{-1}\right)(T)=\varphi(a)(S) T$ $=L(\varphi(a)(S))(T)$. Q.E.D.

LEMMA 1.2. Let $\mathscr{R}$ be a factor with a semifinite, normal trace $\rho$. Let $G$ be a topological group, and let $a \rightarrow \varphi(a)$ be a representation of $G$ into the group of *automorphisms of $\mathscr{R}$. Suppose that $a \rightarrow\langle\varphi(a)(T) x, y\rangle$ is continuous for all $T \in \mathscr{R}$ and $x, y \in \mathscr{H}$. Then there exists a continuous homomorphism $a \rightarrow \lambda(a)$, of $G$ into the positive real numbers, such that $\rho \circ \varphi(a)=\lambda(a) \rho$. 
Proof. $\rho \circ \varphi(a)$ is a semifinite, normal trace on $\mathscr{R}$. Hence $[4$, p. 90 , Corollaire to Théorème 3] there does exist a positive scalar $\lambda(a)$ such that $\rho \circ \varphi(a)=\lambda(a) \rho$. Let $Q$ be a nonzero finite projection in $\mathscr{R}$. Then $T \rightarrow \rho(T Q)$ is a positive normal linear form on $\mathscr{R}$ [4, p. 91, Proposition 5]. Therefore, as $a \rightarrow e$,

$$
0 \leftarrow \rho((\varphi(a)(Q)-Q) Q)=(\lambda(a)-1) \rho\left(Q \varphi\left(a^{-1}\right)(Q)\right)+\rho\left(Q\left(\varphi\left(a^{-1}\right)(Q)-Q\right)\right)
$$

since $\varphi(a)(Q) \rightarrow Q$ in the weak operator topology. But $\rho\left(Q\left(\varphi\left(a^{-1}\right)(Q)-Q\right)\right) \rightarrow 0$ and $\rho\left(Q \varphi\left(a^{-1}\right)(Q)\right) \rightarrow \rho(Q)>0$ as $a \rightarrow 0$. Hence, $\lambda(a) \rightarrow 1$ as $a \rightarrow e$. Q.E.D.

Proposition 1.3. Let $\mathscr{R}$ be an infinite, semifinite factor on the separable Hilbert space $\mathscr{H}$ such that $\mathscr{R}^{\prime}$ is infinite. Let $G$ be a topological group, and let $a \rightarrow \varphi(a)$ be a representation of $G$ into the group of ${ }^{*}$-automorphisms of $\mathscr{R}$. Suppose that $a \rightarrow\langle\varphi(a)(T) x, y\rangle$ is continuous for all $T$ in $\mathscr{R}$ and $x, y$ in $\mathscr{H}$. Then there exists $a$ strongly continuous unitary representation $a \rightarrow U(a)$ of $G$ on $\mathscr{H}$ such that $\varphi(a)(T)$ $=U(a) T U\left(a^{-1}\right)$.

Proof. Let $\rho$ be a semifinite, normal trace on $\mathscr{R}$. Let $\mathscr{A}$ be the algebra over the complex numbers generated by the finite projections in $\mathscr{R}$. If $S, T \in \mathscr{A}$, let $\langle S, T\rangle$ $=\rho\left(T^{*} S\right)$. The pair $\mathscr{A},\langle\cdot, \cdot\rangle$ is a Hilbert algebra. Since $\varphi(a)(P)$ is a finite projection if $P$ is a finite projection, each $\varphi(a)$ defines a ${ }^{*}$-automorphism of $\mathscr{A}$. If $T_{1}$ and $T_{2}$ are in $\mathscr{A}$, then $a \rightarrow \rho\left(T_{2}^{*} \varphi(a)\left(T_{1}\right)\right)$ is continuous on $G$ [4, p. 91, Proposition 5]. Finally, it is known that the mapping $\varphi: T \rightarrow L(T)$ extends to a ${ }^{*}$-isomorphism of $\mathscr{R}$ onto $L(\mathscr{A})$. But this *-isomorphism is spatial since $\mathscr{H}$ is separable and $\mathscr{R}^{\prime}$ is properly infinite [4, p. 321, Corollaire 7]. The theorem now follows from the previous remarks and from Lemma 1.1 , by letting $\mu(a)=\lambda(a)^{-1}$. Q.E.D.

Lemma 1.4. Let $\mathscr{R}$ be a finite factor with trace vector $z$. Let $G$ be a topological group, and let $a \rightarrow \varphi(a)$ be a representation of $G$ into the group of ${ }^{*}$-automorphisms of $\mathscr{R}$. Suppose that $a \rightarrow\langle\varphi(a)(T) x, y\rangle$ is continuous for all $T$ in $\mathscr{R}$ and $x, y$ in $\mathscr{H}$. Then there exists a strongly continuous unitary representation $a \rightarrow U(a)$ of $G$ on $\mathscr{H}$ such that $\varphi(a)(T)=U(a) T U\left(a^{-1}\right)(T \in \mathscr{R})$.

Proof. For $T$ in $\mathscr{R}$, let $U(a)(T z)=\varphi(a)(T) z$. This is a well-defined linear mapping. Furthermore,

$$
\begin{aligned}
\|U(a)(T z)\|^{2} & =\|\varphi(a)(T) z\|^{2} \\
& =\operatorname{trace}\left(\varphi(a)\left(T^{*} T\right)\right) \\
& =\operatorname{trace}\left(T^{*} T\right)=\|T z\|^{2} .
\end{aligned}
$$

Hence, each $U(a)$ extends to an isometry of $\mathscr{H}$, which we again denote by $U(a)$. $U(a)$ is actually a unitary operator since

$$
U(a) U\left(a^{-1}\right)=U\left(a^{-1}\right) U(a)=I .
$$

That $a \rightarrow U(a)$ is strongly continuous follows from the assumption that $a \rightarrow\langle\varphi(a)(T) x, y\rangle$ is continuous. Finally,

$$
U(a) T U\left(a^{-1}\right) z=U(a) T z=\varphi(a)(T) z .
$$

Hence, $\varphi(a)(T)=U(a) T U\left(a^{-1}\right)$ since $z$ is a separating vector $\mathscr{R}$. Q.E.D. 
Proposition 1.5. Let $\mathscr{R}$ be a finite factor on the separable Hilbert space $\mathscr{H}$ such that $\mathscr{R}^{\prime}$ is infinite. Let $G$ be a topological group, and let $a \rightarrow \varphi(a)$ be a representation of $G$ into the group of ${ }^{*}$-automorphisms of $\mathscr{R}$. Suppose that $a \rightarrow\langle\varphi(a)(T) x, y\rangle$ is continuous for all $T$ in $\mathscr{R}$ and $x, y$ in $\mathscr{H}$. Then there exists a strongly continuous unitary representation $a \rightarrow U(a)$ of $G$ on $\mathscr{H}$ such that $\varphi(a)(T)=U(a) T U\left(a^{-1}\right)$ $(T \in \mathscr{R})$.

Proof. There exists a finite factor $\mathscr{S}$ with a trace vector on a Hilbert space $\mathscr{K}$ and a ${ }^{*}$-isomorphism $\psi: \mathscr{R} \rightarrow \mathscr{S}$. Then $a \rightarrow \psi \circ \varphi(a) \circ \psi^{-1}$ is a representation of $G$ into the group of ${ }^{*}$-automorphisms of $\mathscr{S}$, and $a \rightarrow\left\langle\psi \circ \varphi(a) \circ \psi^{-1}(T) x, y\right\rangle$ is continuous for all $T$ in $\mathscr{S}$ and all $x, y$ in the Hilbert space $\mathscr{K}$. By Lemma 1.4 there exists a strongly continuous unitary representation $a \rightarrow W(a)$ of $G$ on $\mathscr{K}$ such that $\psi \circ \varphi(a) \circ \psi^{-1}(T)=W(a) T W\left(a^{-1}\right)(T \in \mathscr{S})$. Let $I$ be the identity on an infinitedimensional separable Hilbert space. Let $\psi^{\prime}(T)=\psi(T) \otimes I(T \in \mathscr{R})$. Then $\psi^{\prime}$ is a *-isomorphism of $\mathscr{R}$ onto $\mathscr{S} \otimes I$, and

$$
\psi^{\prime} \circ \varphi(a) \circ \psi^{\prime-1}(T)=(W(a) \otimes I) T\left(W\left(a^{-1}\right) \otimes I\right) \quad(T \in \mathscr{S} \otimes I) .
$$

But $(\mathscr{S} \otimes I)^{\prime}$ is properly infinite. Hence, $\psi^{\prime}$ is spatial $[4$, p. 321 , Corollaire 7]. Q.E.D.

2. A Borel structure on the spatial *-automorphisms of a von Neumann algebra. Let $\mathscr{S}$ be a von Neumann algebra on the separable Hilbert space $\mathscr{K}$. Throughout this section the following notation will be in effect: $\mathscr{S}_{1}$ is the unit ball of $\mathscr{S}, L(\mathscr{K})$ is the algebra of all bounded operators on $\mathscr{K}, U(\mathscr{S})$ is the unitary group of $\mathscr{S}$, $U(\mathscr{S}, \mathscr{K})$ is the group of unitary operators on $\mathscr{K}$ which induce *-automorphisms of $\mathscr{S}$, and Spaut $(\mathscr{S})$ is the group of spatial *-automorphisms of $\mathscr{S}$. The purpose of this section is to show that Spaut $(\mathscr{S})$ is a standard Borel space with respect to a natural Borel structure, that $U(\mathscr{S}, \mathscr{K})$ is a polonais subgroup of $U(L(\mathscr{K})$ ), and that there exists a Borel cross-section for Spaut $(\mathscr{S})$ in $U(\mathscr{S}, \mathscr{K})$.

LEMMA 2.1. Let $T_{m}(m \geqq 1)$ be a sequence in $\mathscr{S}_{1}$ which is strongly dense in $\mathscr{S}_{1}$. Let $x_{n}(n \geqq 1)$ be a sequence in $\mathscr{K}$ which is strongly dense in $\mathscr{K}$. Let

$$
\mathscr{W}=\bigcap_{j, m, n \geqq 1} \bigcup_{l \geqq 1} \bigcap_{1 \leqq k \leqq n}\left[U \in U(L(\mathscr{K})) \mid\left\|U T_{j} U^{*} x_{k}-T_{l} x_{k}\right\|<\frac{1}{m}\right] .
$$

Then $U(\mathscr{S}, \mathscr{K})=\mathscr{W} \cap \mathscr{W}^{*}$.

Proof. An element $U$ of $U(L(\mathscr{K}))$ is in $U(\mathscr{S}, \mathscr{K})$ if and only if $U \mathscr{P}_{1} U^{*} \subseteq \mathscr{S}_{1}$ and $U^{*} \mathscr{S}_{1} U \subseteq \mathscr{S}_{1}$.

Let $U \in U(\mathscr{S}, \mathscr{K}), j \geqq 1, m \geqq 1$, and $n \geqq 1$. Then $U T_{j} U^{*}$ is in $\mathscr{S}_{1}$. Since the sequence $T_{m}(m \geqq 1)$ is strongly dense in $\mathscr{S}_{1}$, there exists some $T_{l}$ such that

$$
\sup _{1 \leqq k \leqq n}\left\|U T_{j} U^{*} x_{k}-T_{l} x_{k}\right\|<\frac{1}{m} \text {. }
$$


Since this holds for every $j \geqq 1, m \geqq 1$, and $n \geqq 1, U \in \mathscr{W}$. But $U \in U(\mathscr{S}, \mathscr{K})$ implies $U^{*} \in U(\mathscr{S}, \mathscr{K})$. Hence, $U^{*} \in \mathscr{W}$, or $U \in \mathscr{W}^{*}$. Therefore, $U \in \mathscr{W}^{*} \cap \mathscr{W}^{*}$, or $U(\mathscr{S}, \mathscr{K}) \subseteq \mathscr{W} \cap \mathscr{W}^{*}$.

Conversely, suppose that $U \in \mathscr{W} \cap \mathscr{W}^{*}$. We must show that $U \mathscr{S}_{1} U^{*} \subseteq \mathscr{S}_{1}$. It suffices to show that $U T_{j} U^{*} \in \mathscr{S}_{1}$ for all $j \geqq 1$, or, since $\mathscr{S}_{1}$ is strongly closed, that $U T_{j} U^{*}$ lies in the strong closure of $\mathscr{S}_{1}$. But simple estimates show that this last statement holds if, for all $m \geqq 1$ and $n \geqq 1$, there is some $T_{l}$ in

$$
\left[T \in L(\mathscr{K}) \mid \sup _{1 \leqq k \leqq n}\left\|U T_{j} U^{*} x_{k}-T x_{k}\right\|<\frac{1}{m}\right] .
$$

But this holds for all $j \geqq 1, m \geqq 1$, and $n \geqq 1$ if and only if $U$ is in $\mathscr{W}$. Q.E.D.

CoROllaRy 2.2. $U(\mathscr{S}, \mathscr{K})$ is a $G_{\delta}$ in $U(L(\mathscr{K}))$.

Proof. $U(L(\mathscr{K}))$ is a polonais space with respect to the strong operator topology. The corollary now follows from Lemma 2.1. Q.E.D.

COROLlaRY 2.3. $U(\mathscr{S}, \mathscr{K})$ is a polonais space with respect to the strong operator topology.

Proof. This follows from the previous corollary plus [1, Théorème 1, p. 123]. Q:E.D.

$U\left(\mathscr{S}^{\prime}\right)$ is a closed, normal subgroup of $U(\mathscr{S}, \mathscr{K})$. We make the following observation.

LEMMA 2.4. The quotient group $U(\mathscr{S}, \mathscr{K}) / U\left(\mathscr{S}^{\prime}\right)$ is a polonais group.

Let $\pi: U(\mathscr{S}, \mathscr{K}) \rightarrow U(\mathscr{S}, \mathscr{K}) / U\left(\mathscr{S}^{\prime}\right)$ be the natural quotient mapping. $\pi$ is open and continuous.

LEMMA 2.5. There exists a Borel cross-section for the left cosets of $U\left(\mathscr{S}^{\prime}\right)$ in $U(\mathscr{S}, \mathscr{K})$.

Proof. This follows immediately from [3, Lemme 3, p. 279]. Q.E.D.

Let $\psi: U(\mathscr{S}, \mathscr{K}) / U\left(\mathscr{S}^{\prime}\right) \rightarrow$ Spaut $(\mathscr{S})$ be the natural algebraic isomorphism, Endow Spaut $(\mathscr{S})$ with the unique topology and Borel structure such that $\psi$ is a topological and Borel isomorphism. Then Spaut $(\mathscr{S})$ is a polonais space and a standard Borel space.

Lemma 2.6. Let $T \in \mathscr{S}$ and $x, y \in \mathscr{K}$. Then $\varphi(\cdot) \rightarrow\langle\varphi(T) x, y\rangle$ is continuous on Spaut $(\mathscr{S})$.

Proof. The mapping $f: U \rightarrow\left\langle U T U^{*} x, y\right\rangle$, of $U(\mathscr{S}, \mathscr{K}) \rightarrow C^{1}$, is continuous on $U(\mathscr{S}, \mathscr{K})$. Since $\left\langle U T U^{*} x, y\right\rangle=\left\langle W T W^{*} x, y\right\rangle$ if $U$ and $W$ lie in the same $U\left(\mathscr{S}^{\prime}\right)$ coset in $U(\mathscr{S}, \mathscr{K}), f$ gives rise to a continuous mapping,

$$
g: U(\mathscr{S}, \mathscr{K}) / U\left(\mathscr{S}^{\prime}\right) \rightarrow C^{1}
$$

where $g(\pi(U))=\left\langle U T U^{*} x, y\right\rangle$. But the mapping $\varphi(\cdot) \rightarrow\langle\varphi(T) x, y\rangle$ is just $g \circ \psi^{-1}(\varphi)$, and hence is continuous. Q.E.D. 
Proposition 2.7. The Borel structure on Spaut $(\mathscr{S})$ is the smallest such that the mappings $\varphi(\cdot) \rightarrow\langle\varphi(T) x, y\rangle(T$ in $\mathscr{S}$ and $x, y$ in $\mathscr{K})$ are Borel.

Proof. Let $\mathscr{B}_{1}$ be our original Borel structure on Spaut $(\mathscr{S})$ and let $\mathscr{B}_{2}$ be the Borel structure on Spaut $(\mathscr{S})$ generated by the mappings $\varphi(\cdot) \rightarrow\langle\varphi(T) x, y\rangle$.

$\mathscr{B}_{1} \supseteq \mathscr{B}_{2}$ by Lemma 2.6. But $\mathscr{B}_{1}$ is standard and $\mathscr{B}_{2}$ is obviously countably separated. Hence, $\mathscr{B}_{1}=\mathscr{B}_{2}$ by [7, Theorem 3.3, p. 139]. Q.E.D.

3. Some direct integral theory. Let $\mathscr{R}$ be a von Neumann algebra on the separable Hilbert space $\mathscr{H}$. Recall that there exists a standard Borel space $\Xi$, a $\sigma$-finite Borel measure $\mu$ on $\Xi$, a separable Hilbert space $\mathscr{K}$, and a Borel field $\xi \rightarrow \mathscr{R}(\xi)$ of factors on $\mathscr{K}$ such that $\mathscr{R}=\int_{\Xi}^{\oplus} \mathscr{R}(\xi) d \mu(\xi)$ and Cent $\mathscr{R}=L^{\infty}(\Xi, \mu)$. This setup describes the central, direct integral decomposition of $\mathscr{R}$. Using this notation we prove the following theorem in this section.

THEOREM 3.1. Let $G$ be a separable locally compact group, and let $a \rightarrow \varphi(a)$ be $a$ representation of $G$ into the group of spatial, center-fixing *automorphisms of $\mathscr{R}$ such that $a \rightarrow\langle\varphi(a)(T) x, y\rangle$ is continuous for all $T$ in $\mathscr{R}$ and $x, y$ in $\mathscr{H}$. Then there exists a Borel set $\Xi^{\prime} \subset \Xi$ with $\mu\left(\Xi-\Xi^{\prime}\right)=0$ such that for $\xi \in \Xi^{\prime}$, there exists a representation $a \rightarrow \varphi_{\xi}(a)$ of $G$ into the group of *automorphisms of $\mathscr{R}(\xi)$ such that $a \rightarrow\left\langle\varphi_{\xi}(a)(T) x, y\right\rangle$ is continuous for all $T$ in $\mathscr{R}(\xi)$ and $x, y$ in $\mathscr{K}$.

Many of the techniques used in this section are the same or similar to those used in [11].

The proof of the following lemma is close to the proof of Theorem B, p. 68, of [5].

Lemma 3.2. Let $G$ be a separable locally compact group, and let $E \subseteq G$ be a Borel measurable subset of $G$ of positive, finite measure. Then $E \cdot E^{-1}$ contains a neighborhood of the identity.

Proof. Let $\mu$ be left invariant Haar measure on $G . a \rightarrow \mu(E \cup a \cdot E)$ is continuous on $G$. Hence, the set $V$ of $a$ such that $\mu(E \cup a \cdot E)<2 \mu(E)$ is a neighborhood of $e$ in $G$. Now if $a$ is in $V$, then $E \cap a \cdot E \neq \varnothing$, for if this intersection were empty, then $\mu(E \cup a \cdot E)=\mu(E)+\mu(a \cdot E)=2 \mu(E)$. Contradiction. Hence, $V \subseteq E \cdot E^{-1}$. Q.E.D.

COROLlary 3.3. Let $G$ be a separable locally compact group, and let $E \subseteq G$ be a Borel subset of $G$ which differs from $G$ on a Borel null set. Then the group (algebraically) generated by $E$ equals $G$.

Proof. Since $E$ differs from $G$ only on a null set, $E$ is dense in $G$. By Lemma 3.2, this subgroup is open, and hence equals $G$. Q.E.D.

COROLlARY 3.4. Let $\mathscr{B}$ be a $C^{*}$-algebra on the Hilbert space $\mathscr{K}$ which generates the von Neumann algebra $\mathscr{S}$. Suppose that $a \rightarrow \varphi(a)$ is a representation of the separable locally compact $G$ into the group of *automorphisms of $\mathscr{B}$. Suppose there exists a Borel subset $E$ of $G$, which differs from $G$ on a Borel null set, such that a in E implies $\varphi(a)$ extends to $a^{*}$-automorphism of $\mathscr{S}$. Then each $\varphi(a)$ extends to a *-automorphism of $\mathscr{S}$. 
Proof. The set $D$ of elements of $G$ which extend to *-automorphisms of $\mathscr{S}$ clearly is a subgroup of $G$ which contains $E$. But by Corollary 3.3, $E$ algebraically generates $G$. Hence, $D=G$. Q.E.D.

Lemma 3.5. Let $\mathscr{R}, G$, and $a \rightarrow \varphi(a)$ be as in the hypotheses of Theorem 3.1. Then there is a separable $C^{*}$-algebra $\mathscr{B}$ in $\mathscr{R}$ such that

(1) $\mathscr{B}$ is dense in $\mathscr{R}$ in the strong operator topology;

(2) $\mathscr{B}$ is invariant under each $\varphi(a)$;

(3) if $T$ is in $\mathscr{B}$, then $\|\varphi(a)(T)-T\| \rightarrow 0($ as $a \rightarrow e)$;

(4) $\mathscr{B} \cap$ Cent $\mathscr{R}$ is strongly dense in Cent $\mathscr{R}$.

Proof. Let $T(i)(i \geqq 1)$ be strongly dense in Cent $\mathscr{R}$. Let $f(j)(j \geqq 1)$ be a sequence of continuous functions with compact support which are dense in $L^{1}(G)$. Let $S(k)(k \geqq 1)$ be a sequence in $\mathscr{R}$ which is strongly dense in $\mathscr{R}$. Take $\mathscr{B}$ to be the $C^{*}$-algebra generated by $T(i)(i \geqq 1)$ and $\int_{G} f(j)(a) \varphi(a)(S(k)) d a(j, k \geqq 1) . \mathscr{B}$ is a separable $C^{*}$-subalgebra of $\mathscr{R}$ and (1)-(4) are immediate. Q.E.D.

We will be working with this $C^{*}$-algebra $\mathscr{B}$ from now on. Without any loss of generality, we make the following assumptions. Given $T$ in $\mathscr{B}$, there exists a Borel mapping $\xi \rightarrow T(\xi)$ of $\Xi$ into $L(\mathscr{K})$ such that $T=\int \oplus T(\xi) d \mu(\xi) ;\|T(\xi)\| \leqq\|T\|$ for each $\xi$ in $\Xi: T \rightarrow T(\xi)$ is a representation of $\mathscr{B}$ on $\mathscr{K}$, for each $\xi$ in $\Xi$; the von Neumann algebra generated by the $T(\xi)(T$ in $\mathscr{B})$ is $\mathscr{R}(\xi)$, for each $\xi$ in $\Xi$.

If $E$ is a subset of a set $S, I(E)$ will denote the indicator function of $E$.

LEMMA 3.6. Suppose that we have a mapping $(a, \xi) \rightarrow U(a, \xi)$ of $G \times \Xi \rightarrow U(L(\mathscr{K}))$ such that

(1) $\xi \rightarrow U(a, \xi)$ is a Borel function for each fixed $a$;

(2) $a \rightarrow \int_{\Xi}\langle U(a, \xi) x, y\rangle I(E)(\xi) d \mu(\xi)$ is a Borel function on $G$, for each $x, y$ in $\mathscr{K}$ and Borel set $E$ in $\Xi$ with $\mu(E)<+\infty$. Then there exists a Borel mapping $(a, \xi) \rightarrow W(a, \xi)$ of $G \times \Xi \rightarrow U(L(\mathscr{K}))$ such that, except for possibly a Borel null set, $U(a, \xi)=W(a, \xi)$ for $\mu$-almost all $\xi$.

Proof. Choose an orthonormal basis $x(1), x(2), \ldots$ of $\mathscr{K}$. Apply Lemma 3.1 of [9] to the functions $\langle U(a, \xi) x(i), x(j)\rangle$. We obtain that there exist Borel functions $m_{i j}(a, \xi)$ on $G \times \Xi$ such that for all $i, j \geqq 1$ and almost every $a$,

$$
\langle U(a, \xi) x(i), x(j)\rangle=m_{i j}(a, \xi)
$$

for $\mu$-almost every $\xi$. Then there exists a null Borel subset $N$ of $G \times \Xi$ such that if $(a, \xi)$ is in $G \times \Xi-N$, the matrix $\left(m_{i j}(a, \xi)\right)$ represents a unitary operator $W^{\prime}(a, \xi)$. Let $W(a, \xi)=W^{\prime}(a, \xi)$ for $(a, \xi)$ in $G \times \Xi-N$ and let $W(a, \xi)=I$ for $(a, \xi)$ in $N$. Then $W(a, \xi)$ is a unitary operator for each $(a, \xi)$ in $G \times \Xi$ and $(a, \xi) \rightarrow W(a, \xi)$ is a Borel mapping. One may easily check that except for possibly a Borel null set, $U(a, \xi)=W(a, \xi)$ for $\mu$-almost all $\xi$. Q.E.D.

Now any center-fixing automorphism of a von Neumann algebra on a separable Hilbert space, whose commutant has no finite portion, is spatial [4, Corollaire 8, p. 322]. Hence, by the results of $\S 2$, there exists a Borel mapping $a \rightarrow U(a)$ of $G$ 
into $U(\mathscr{R})$ such that $\varphi(a)(T)=U(a) T U(a)^{*}$ for all $a$ in $G$ and $T$ in $\mathscr{R}$. Now each $U(a)$ is in $(\text { Cent } \mathscr{R})^{\prime}$. Hence, for each $U(a)$ there exists a Borel field $\xi \rightarrow U(a, \xi)$ of unitaries such that

$$
U(a)=\int_{\Xi}^{\oplus} U(a, \xi) d \mu(\xi) .
$$

For any $x, y$ in $\mathscr{K}$ and any Borel subset $E$ of $\Xi$ of finite positive measure, $\int_{\Xi}\langle U(a, \xi) x, y\rangle I(E)(\xi) d \mu(\xi)$ is a Borel function of $a$.

Hence, we can conclude that there exists a Borel mapping $(a, \xi) \rightarrow W(a, \xi)$ of $G \times \Xi \rightarrow U(L(\mathscr{K}))$ such that $\int_{\Xi}^{\oplus} W(a, \xi) d \mu(\xi)$ induces $\varphi(a)$ except for a null set of $a$.

LEMMA 3.7. If $a$ is in $G$, there is a Borel null set $N(a)$ of $\Xi$ such that for $\xi \in \Xi-N(a), T(\xi) \rightarrow(\varphi(a)(T))(\xi)$ is well defined and is $a *^{*}$-automorphism of $\mathscr{B}(\xi)$.

Proof. Choose a sequence $T(j)(j \geqq 1)$ which is dense in $\mathscr{B}$. Except for at most a Borel null set $N(j)$,

$$
(\varphi(a)(T(j)))(\xi)=W(a, \xi) T(j)(\xi) W(a, \xi)^{*}
$$

and

$$
\left(\varphi\left(a^{-1}\right)(T(j))\right)(\xi)=W(a, \xi)^{*} T(j)(\xi) W(a, \xi) .
$$

Let $N(a)=\bigcup_{j \geqq 1} N(j)$. Therefore, for $\xi$ in $\Xi-N(a)$,

$$
W(a, \xi) \mathscr{B}(\xi) W(a, \xi)^{*} \subseteq \mathscr{B}(\xi), \quad W(a, \xi)^{*} \mathscr{B}(\xi) W(a, \xi) \subseteq \mathscr{B}(\xi),
$$

and

$$
(\varphi(a)(T))(\xi)=W(a, \xi) T(\xi) W(a, \xi) . \quad \text { Q.E.D. }
$$

Lemma 3.8. There exists a Borel null set $N$ in $\Xi$ such that for $\xi \in \Xi-N$, $\varphi_{\xi}(a): T(\xi) \rightarrow(\varphi(a)(T))(\xi)$ is well defined and is $a^{*}$-automorphism of $\mathscr{B}(\xi)$ for each $a$ in $G$.

Proof. Choose a dense sequence $a_{k}$ in $G$, let $N\left(a_{k}\right)$ be as in the previous lemma, and let $N=\bigcup_{k \geqq 1} N\left(a_{k}\right)$.

$\varphi_{\xi}(a)$ is well defined for $\xi \in \Xi-N$. For suppose that $T(\xi)=0$. Then $\left(\varphi\left(a_{k}\right)(T)\right)(\xi)=0$ for all $k \geqq 1$. Let $a_{k_{l}}$ converge to $a$. Then

$$
\begin{aligned}
\|(\varphi(a)(T))(\xi)\| & =\left\|(\varphi(a)(T))(\xi)-\left(\varphi\left(a_{k_{l}}\right)(T)\right)(\xi)\right\| \\
& \leqq\left\|\varphi(a)(T)-\varphi\left(a_{k_{l}}\right)(T)\right\| \rightarrow 0 \quad(\text { as } l \uparrow+\infty) .
\end{aligned}
$$

Hence, $(\varphi(a)(T))(\xi)=0$. Hence, $\varphi_{\xi}(a)$ is well defined for each element of $G$. It is clear that $\varphi_{\xi}(a)$ is a ${ }^{*}$-homomorphism of $\mathscr{B}(\xi)$ into itself. $\varphi_{\xi}(a)$ is a ${ }^{*}$-automorphism since $\varphi_{\xi}\left(a^{-1}\right)$ is its inverse. Q.E.D.

In what follows, we toss out the Borel null set $N$. We may do this since $\Xi-N$ is again a standard Borel space.

Lemma 3.9. Fix $x$ and $y$ in $\mathscr{K}$ and $T$ in $\mathscr{B}$. Then $(a, \xi) \rightarrow\langle(\varphi(a)(T))(\xi) x, y\rangle$ is $a$ Borel mapping. 
Proof. For each fixed $a, \xi \rightarrow\langle(\varphi(a)(T))(\xi) x, y\rangle$ is a Borel mapping. For each fixed $\xi, a \rightarrow\langle(\varphi(a)(T))(\xi) x, y\rangle$ is continuous. The lemma now follows from [8, Lemma 9.2, p. 122]. Q.E.D.

LEMMA 3.10. The set of $(a, \xi)$ such that $W(a, \xi)$ induces $\varphi_{\xi}(a)$ is a Borel subset of $G \times \Xi$.

Proof. For any $T$ in $\mathscr{B}$ and $x, y$ in $\mathscr{K}$,

$$
(a, \xi) \rightarrow\left\langle W(a, \xi) T(\xi) W(a, \xi)^{*} x, y\right\rangle-\langle(\varphi(a)(T))(\xi) x, y\rangle
$$

is a Borel mapping. This follows from the previous lemma plus the fact that $(a, \xi) \rightarrow W(a, \xi)$ is a Borel mapping. Hence, if $x(i)(i \geqq 1)$ is a dense sequence in $\mathscr{K}$ and $T(j)(j \geqq 1)$ is a dense sequence in $\mathscr{B}$,

$$
\bigcap_{i, j, k \geqq 1}\left[(a, \xi) \mid\left\langle W(a, \xi) T(i)(\xi) W(a, \xi)^{*} x(j), x(k)\right\rangle=\langle\varphi(a)(T(i))(\xi) x(j), x(k)\rangle\right]
$$

is a Borel set. But this set is just the set of $(a, \xi)$ such that $W(a, \xi)$ induces $\varphi_{\xi}(a)$. Q.E.D.

Lemma 3.11. Except for at most a Borel null set of $\xi, \varphi_{\xi}(a)$ extends to $a *_{\text {- }}$ automorphism of $\mathscr{R}(\xi)$ for every $a$ in $G$.

Proof. Except for at most a null Borel set, $W(a, \xi)$ induces $\varphi_{\xi}(a)$ for $\mu$-almost every $\xi$. Fubini's theorem and Lemma 3.10 implies that there exists a null Borel subset $N(1)$ of $G \times \Xi$ such that $(a, \xi)$ in $G \times \Xi-N(1)$ implies that $W(a, \xi)$ induces $\varphi_{\xi}(a)$. Fubini's theorem again implies that there exists a Borel null set $N(2)$ of $\Xi$ such that $\xi$ in $\Xi-N(2)$ implies that $W(a, \xi)$ induces $\varphi_{\xi}(a)$ for almost every $a$. Therefore, Corollary 3.4 implies that $\varphi_{\xi}(a)$ is extendable to a *automorphism of $\mathscr{R}(\xi)$ for every $a$ and every $\xi$ in $\Xi-N(2)$. Q.E.D.

In what follows, toss out the Borel null set $N(2)$. We may do this since $\Xi-N(2)$ is again a standard Borel space.

Lemma 3.12. $a \rightarrow\left\langle\varphi_{\xi}(a)(T) x, y\right\rangle$ is continuous for all $T$ in $\mathscr{R}(\xi)$ and $x, y$ in $\mathscr{K}$.

Proof. If $T=S(\xi)$, where $S$ is in $\mathscr{B}$, then

$$
\begin{aligned}
&\left|\left\langle\varphi_{\xi}(a)(T) x, y\right\rangle-\left\langle\varphi_{\xi}(b)(T) x, y\right\rangle\right| \leqq\left\|\varphi_{\xi}(a)(S(\xi))-\varphi_{\xi}(b)(S(\xi))\right\| \cdot\|x\| \cdot\|y\| \\
& \leqq\|\varphi(a)(S)-\varphi(b)(S)\| \cdot\|x\| \cdot\|y\| \rightarrow 0 \\
& \text { as } b \rightarrow a .
\end{aligned}
$$

The lemma may now be concluded from the main theorem of [12]. Q.E.D.

4. Proof of Theorem 0.1. We start by noting the following lemma.

LEMMA 4.1. Let $\mathscr{R}=\int_{\Xi}^{\oplus} \mathscr{R}(\xi) d \mu(\xi)$ be the central decomposition of $\mathscr{R}$, as in $\S 3$. Then there exists a Borel null set $N \subseteq \Xi$ such that if $\xi \in \Xi-N$, then $\mathscr{R}(\xi)$ is a semifinite factor and $\mathscr{R}(\xi)^{\prime}$ is infinite. 
Proof. $\xi \rightarrow \mathscr{R}(\xi)^{\prime}$ is a measurable family of von Neumann algebras, and $\mathscr{R}^{\prime}=\int_{\Xi}^{\oplus} \mathscr{R}(\xi)^{\prime} d \mu(\xi)$ is the central decomposition of $\mathscr{R}^{\prime}$. There exists a Borel null set $N(1)$ in $\Xi$ such that $\xi \in \Xi-N(1)$ implies that $\mathscr{R}(\xi)^{\prime}$ is infinite [4, Théorème 2, p. 211]. There exists another null Borel set $N(2)$ in $\Xi$ such that $\xi \in \Xi-N(2)$ implies $\mathscr{R}(\xi)$ is semifinite. Let $N=N(1) \cup N(2)$. Q.E.D.

We toss out the null set $N$. We may do this since $\Xi-N$ is still a standard Borel space.

Let $\mathscr{B}$ and $\varphi_{\zeta}(\cdot)$ be as in $\S 3$.

Lemma 4.2. $\xi \rightarrow\left\langle\varphi_{\xi}(a)(T(\xi)) x, y\right\rangle$ is a Borel function on $\Xi$ for each $a$ in $G, T$ in $\mathscr{B}$, and $x, y$ in $\mathscr{K}$.

Proof. For each $s$ in $\mathscr{B}$ and $x, y$ in $\mathscr{K}, \xi \rightarrow\langle S(\xi) x, y\rangle$ is a Borel function on $\Xi$. If $T$ is in $\mathscr{B}$, then $\varphi(a)(T)$ is also in $\mathscr{B}$ and $\varphi(a)(T)(\xi)=\varphi_{\xi}(a)(T(\xi))$. Q.E.D.

Let $\operatorname{Rep}(G)$ be the set of strongly continuous unitary representations of $G$ on $\mathscr{K}$. $\operatorname{Rep}(G)$ has a natural topology which makes it into a polonais space [2, pp. 315-316]. In this topology $U_{\alpha}(\cdot) \rightarrow U(\cdot)$ if and only if

$$
\sup _{a \in C}\left\|U_{\alpha}(a) x-U(a) x\right\| \rightarrow 0
$$

for each $x \in \mathscr{K}$ and compact subset $C$ of $G$. As the reader may easily check, for each $a \in G$, the mapping $U(\cdot) \rightarrow U(a)$, of $\operatorname{Rep}(G)$ into $U(L(\mathscr{K}))$, is continuous.

The following lemma is a slight extension and modification of [8, Lemma 9.2, p. 122].

LeMmA 4.3. Let $S_{1}$ be a Borel space and $S_{2}$ a separable metric space. Let $f: S_{1} \times S_{2} \rightarrow C^{\prime}$ be such that $s_{1} \rightarrow f\left(s_{1}, s_{2}\right)$ is a Borel function for each $s_{2} \in S_{2}$, and $s_{2} \rightarrow f\left(s_{1}, s_{2}\right)$ is continuous for each fixed $s_{1} \in S_{1}$. Then $f$ is a Borel function on $S_{1} \times S_{2}$.

Proof. Let $t_{n}(m \geqq 1)$ be a dense sequence in $S_{2}$. Let $B\left(t_{n}, \varepsilon\right)$ be the closed ball of radius $\varepsilon$ about $t_{n}$. For $m \geqq 1$, inductively define

$$
\begin{aligned}
C(1, m) & =B\left(t_{1}, 1 / m\right), \\
C(k+1, m) & =B\left(t_{k+1}, 1 / m\right)-\bigcap_{1 \leqq i \leqq k}\left(B\left(t_{k+1}, 1 / m\right) \cap C(i, m)\right) .
\end{aligned}
$$

Then $C(j, m) \cap C(k, m)=\varnothing$ if $j \neq k$, and $\bigcup_{k \geqq 1} C(k, m)=S_{2}$. Define $f_{m}\left(s_{1}, s_{2}\right)$ $=\sum_{k \geqq 1} f\left(s_{1}, t_{k}\right) I(C(k, m))\left(s_{2}\right)$. One checks easily that $f_{m}(m \geqq 1)$ is a Borel function on $S_{1} \times S_{2}$, and $f_{m}\left(s_{1}, s_{2}\right) \rightarrow f\left(s_{1}, s_{2}\right)($ as $m \uparrow+\infty)$ for each $\left(s_{1}, s_{2}\right) \in S_{1} \times S_{2}$. Hence, $f$ is a Borel function on $S_{1} \times S_{2}$. Q.E.D.

Lemma 4.4. Let $A=\left[(\xi, U(\cdot)) \in \Xi \times \operatorname{Rep}(G) \mid U(\cdot)\right.$ induces $\varphi_{\xi}(\cdot)$ on $\left.\mathscr{R}(\xi)\right]$. Then $A$ is a Borel subset of $\Xi \times \operatorname{Rep}(G)$.

Proof. Let $a_{k}(k \geqq 1)$ be a dense sequence in $G$, let $T_{l}(l \geqq 1)$ be a dense sequence in $\mathscr{B}$, and let $x_{m}(m \geqq 1)$ be a dense sequence in $\mathscr{K}$. Each of the mappings

$$
(\xi, U(\cdot)) \rightarrow\left\langle\left[U(a) T(\xi) U\left(a^{-1}\right)-\varphi_{\xi}(a)(T(\xi))\right] x_{m}, x_{n}\right\rangle
$$


is a Borel function on $\Xi$, for each fixed $U(\cdot) \in \operatorname{Rep}(G)$, and is continuous on $\operatorname{Rep}(G)$, for each fixed $\xi \in \Xi$. Hence, Lemma 4.3 implies that each of these functions is a Borel function on $\Xi \times \operatorname{Rep}(G)$. But

$$
A=\bigcap_{k, l, m, n \geqq 1}\left[(\xi, U(\cdot)) \mid\left\langle\left[U\left(a_{k}\right) T_{l}(\xi) U\left(a_{k}^{-1}\right)-\varphi_{\xi}\left(a_{k}\right)\left(T_{l}(\xi)\right)\right] x_{m}, x_{n}\right\rangle=0\right] .
$$

Hence, $A$ is a Borel subset of $\Xi \times \operatorname{Rep}(G)$. Q.E.D.

Proof of Theorem 0.1. Let $A$ be as in the previous lemma. Combining the results of $\S 3$ with Lemma 3.1, we see that $A \cap(\xi \times \operatorname{Rep}(G))$ is nonempty for each $\xi \in \Xi$. But by [7, Theorem 6.3, p. 143], there exists a null Borel set $N$ in $\Xi$ and a Borel mapping, $\xi \rightarrow U_{\xi}(\cdot)$, of $\Xi-N$ to $\operatorname{Rep}(G)$, such that $\left(\xi, U_{\xi}(\cdot)\right) \in A$ for each $\xi \in \Xi-N$. Let $U(a)=\int_{\Xi}^{\oplus} U_{\xi}(a) d \mu(\xi)$. Then $a \rightarrow U(a)$ is a strongly continuous unitary representation of $G$ on $\mathscr{H}$, and $U(a)$ induces $\varphi(a)$ for each $a$ in $G$. Q.E.D.

Remark added in proof. $M$. Henle has given a very elegant proof of the Type III analogue of Theorem 0.1 in his 1970 Yale thesis.

\section{BIBLIOGRAPHY}

1. N. Bourbaki, Topologie générale, Chapitre 9, 2nd ed., Hermann, Paris, 1958.

2. J. Dixmier, Les $C^{*}$-algèbres et leurs représentations, Cahiers Scientifiques, fasc. 29 , Gauthier-Villars, Paris, 1964. MR 30 \#1404.

3. - Dual et quasi-dual d'une algèbre de Banach involutive, Trans. Amer. Math. Soc. 104 (1962), 278-283. MR 25 \#3384.

4. —_Les algèbres d'opérateurs dans l'espace Hilbertien (Algèbres de von Neumann), Cahiers Scientifiques, fasc. 25, Gauthier-Villars, Paris, 1957. MR 20 \#1234.

5. P. R. Halmos, Measure theory, Van Nostrand, Princeton, N. J., 1950. MR 11, 504.

6. R. V. Kadison, Isomorphisms of factors of infinite type, Canad. J. Math. 7 (1955), 322-327. MR 17, 178.

7. G. W. Mackey, Borel structure in groups and their duals, Trans. Amer. Math. Soc. 85 (1957), 134-165. MR 19, 752.

8. - Induced representations of locally compact groups. I, Ann. of Math. (2) 55 (1952), 101-139. MR 13, 434.

9. - A theorem of Stone and von Neumann, Duke Math. J. 16 (1949), 313-326. MR 11, 10.

10. K. R. Parthasarathy, Probability measures on metric spaces, Probability and Math. Statist., no. 3, Academic Press, New York, 1967. MR 37 \#2271.

11. R. R. Kallman, Groups of inner automorphisms of von Neumann algebras, J. Functional Analysis (to appear).

12. —, A remark on a theorem of J. F. Aarnes, Comm. Math. Phys. 14 (1969), 13-14.

YALE UNIVERSITY,

New Haven, Connecticut 06520 